(c) American Dairy Science Association, 2006.

\title{
Risk Factors Associated with Cryptosporidium Infection on Dairy Farms in a New York State Watershed
}

\author{
S. R. Starkey, K. R. Kimber, S. E. Wade, S. L. Schaaf, M. E. White, and H. O. Mohammed ${ }^{\mathbf{1}}$ \\ Department of Population Medicine and Diagnostic Sciences, College of Veterinary Medicine, Cornell University, Ithaca, NY 14853
}

\begin{abstract}
A cross-sectional study was carried out to determine the prevalence of Cryptosporidium parvum-like oocyst shedding on dairy farms in a watershed in New York State and to identify the factors that put animals at risk. A proportional sample of dairy herds in the targeted area was obtained, and animals were selected using a stratified sampling design to ensure representation of the population at risk. Fecal samples were collected per rectum and analyzed for the presence of $C$. parvum-like oocysts using the quantitative centrifugation concentration flotation technique and a proprietary enzyme-linked immunoassay. Additionally, isolates of Cryptosporidium were examined via bidirectional DNA sequencing. Data on putative risk factors were collected at the time of sampling and analyzed for association using logistic regression. The herd prevalence was $42 \%$ and the overall animal prevalence was $3.2 \%$. The prevalence among animals less than $60 \mathrm{~d}$ of age was $20 \%$. The likelihood of shedding Cryptosporidium decreased with the age of the animal and varied with the type of barn water source. Both the number of unweaned calves present at the time of the study, and whether the calves were tied vs. not tied increased the risk of infection. There was significant agreement between the flotation and PCR techniques. Sequencing revealed that $50 \%$ of the isolates were Cryptosporidium bovis, an isolate thought to be nonzoonotic.
\end{abstract}

Key words: prevalence, cross-sectional, Cryptosporidium, risk factor

\section{INTRODUCTION}

Since members of the genus Cryptosporidium were first found to infect humans and animals over $30 \mathrm{yr}$ ago, extensive research has been conducted on these protozoa (Panciera et al., 1971; Navin and Juranek, 1984). Epidemiologic studies have shown that the majority of human disease attributable to members of the

Received January 27, 2006.

Accepted May 8, 2006.

${ }^{1}$ Corresponding author: hom1@cornell.edu genus results from the species Cryptosporidium parvum and Cryptosporidium hominis (Xiao and Ryan, 2004). Although C. parvum has been isolated from multiple mammalian species, including cattle, $C$. hominis is believed to be an exclusively human pathogen (Hunter and Thompson, 2005). Infections in humans typically cause a self-limiting disease of 7 to $10 \mathrm{~d}$ duration, characterized by diarrhea with associated vomiting and abdominal discomfort. However, among young, elderly, and immunocompromised individuals, the condition can become severe and potentially life threatening (Current et al., 1983; Nannini and Okhuysen 2002; Tumwine et al., 2005). The introduction of antiretroviral therapy has reduced the impact of Cryptosporidium among many HIV patients in developed nations (Maggi et al., 2000).

Transmission of Cryptosporidium species takes place via the fecal-oral route, and susceptible hosts contract the infection by ingesting the infective form of the parasite, the sporulated oocyst. Human cryptosporidiosis is most often associated with the consumption of contaminated drinking water or exposure to contaminated recreational water, such as public swimming pools. A small number of cases have also been attributed to contaminated foods such as salads (Fayer et al., 2000).

In cattle, $C$. parvum typically causes disease in calves and has been identified as one of the primary etiologic agents of neonatal calf diarrhea (Naciri et al., 1999). Herd-level prevalence of $C$. parvum has been reported to range from 13 to 100\% (Wade et al., 2000; Santin et al., 2004), although a portion of this variability is likely attributable to study design and the geographical region under investigation. A new species, Cryptosporidium bovis, has recently been described based on both genomic and host range differences (Fayer et al., 2005). To date, this organism has been identified only in cattle and is thus considered unlikely to be zoonotic. Cryptosporidium bovis is indistinguishable from C. parvum using traditional diagnostic methods such as flotation and ELISA techniques; thus, the term "C. parvum-like" has been used to describe infections identified using such methods (Starkey et al., 2005).

Research efforts were increased after Cryptosporidium species were found to be responsible for several 
large waterborne disease outbreaks in North America, the United Kingdom, and Europe (Patel et al., 1998; Ong et al., 1999; Glaberman et al., 2002). The largest such outbreak occurred in Milwaukee in 1993 (Mac Kenzie et al., 1994). In their 1994 paper, Mac Kenzie et al. speculated that the source of Cryptosporidium oocysts may have been contamination of the body of water from which a failed water treatment plant drew its intake-Milwaukee harbor-by cattle herds or slaughterhouses located along 2 rivers leading to the harbor. In addition to animal sources, the authors also commented on the possible role of human sewage and mixed-source runoff attributable to spring rains and thawing snow. Subsequent molecular work indicated that $C$. hominis was responsible for the Milwaukee outbreak (Sulaiman et al., 2001). This finding essentially removed cattle from consideration as the source of the outbreak because $C$. hominis has never been isolated from cattle, and it is considered an anthroponotic pathogen (Morgan-Ryan et al., 2002). However, C. parvum has been identified at the molecular level in a number of smaller waterborne outbreaks (Ong et al., 1999; Glaberman et al., 2002). This finding, along with the fact that cattle are quite common within watersheds in the United States, has led to further speculation about their role in the contamination of drinking water supplies (Xiao and Ryan, 2004). Furthermore, human dose-response studies have shown that $C$. parvum of bovine origin can have an infectious dose $50\left(\mathrm{ID}_{50}\right)$ as low as 87 oocysts (Okhuysen et al., 1999). Therefore, there is little doubt that cattle pose a potential, albeit imperfectly quantified, risk to human health with regard to C. parvum. An additional source of this imperfect risk quantification stems from the relative lack of information about the prevalence of the nonzoonotic $C$. bovis, especially among cattle populations within watersheds.

Dairy farms represent a significant industry within New York State, with cash receipts from milk totaling over $\$ 1.56$ billion in 2002 , more than half of all agricultural receipts collected that year (New York State Department of Agriculture and Markets, 2006). Such operations are often located within the prime agricultural lands that form watersheds. Academic institutions as well as state and local government agencies have conducted extensive research on the dynamics of infection of Cryptosporidium in dairy cattle as a means to better understand the level of risk these animals pose to the environment. By way of continuation of such research activities, the present study aimed to determine the prevalence of $C$. parvum in the Upper Susquehanna Watershed and investigate the potential risk factors associated with the prevalence of C. parvum (as identified by traditional methods) in the target population. Additionally, the potential role of cattle in the risk to water quality was examined by differentiating between zoonotic and nonzoonotic isolates using DNA sequencing.

\section{MATERIALS AND METHODS}

\section{Study Design}

The target population consisted of all cattle on dairy farms within the approximately 275 -square-mile portion of the Upper Susquehanna River Watershed located in Delaware County, New York. This region spans the northern border of the county and covers approximately $20 \%$ of the county's 1,460 square miles. A crosssectional study was designed and conducted over a 3mo period (June to August). Sampling was conducted over this relatively short time period to reduce any potential confounding effects of season on the prevalence of Cryptosporidium.

The study population consisted of 19 dairy farms selected from the target population of approximately 100 farms. A block sampling design was adopted to ensure representation of all geographic areas covered by the target population. In this design, farms were grouped into clusters according to their geographic location within the county (i.e., towns). Within this framework, a proportional sampling scheme was also used. Thus, although all areas were represented among the selected farms, more study farms were included from those areas with a relatively higher proportion of dairy operations. While following the above study design, we used a participatory approach for the selection of study farms. This approach involved collaboration with the Delaware County Soil and Water Conservation District and representatives of the local farmers' association. The collaborators provided planning and logistical support and facilitated study farm selection by granting access to their ongoing, countywide farm census data. In addition to providing census data, the collaborators also provided detailed local knowledge during the study farm selection phase. We believe that the participatory approach, in conjunction with the proportional block design, led to the selection of a representative study population.

\section{Sample and Data Collection}

An age-stratified sampling design was used to collect fecal samples on the study farms (Wade et al., 2000; Santin et al., 2004). Animals less than 6 mo of age were differentially targeted to improve the chances of detecting those shedding $C$. parvum-like oocysts. In the present study, the fecal sampling protocol called for the sampling on study farms of all calves less than 6 mo of age, up to a maximum of 15 animals. If more than 15 
Table 1. Summary of variables from dairy farm interviews used in data analysis

\begin{tabular}{|c|c|}
\hline Variable (risk factor) & Description \\
\hline Source of barn water & Categorical: Well, other \\
\hline Barn type & Categorical: Free stall, tie stall \\
\hline Herd size increased by $10 \%$ or more in past year & Categorical: Yes, no \\
\hline Herd size decreased by $10 \%$ or more in past year & Categorical: Yes, no \\
\hline Milkers allowed access to pasture in summer & Categorical: Yes, no \\
\hline Rodent control on farm & Categorical: Present or absent \\
\hline \multicolumn{2}{|l|}{ Maternity management } \\
\hline Dedicated calving area & Categorical: Yes, no \\
\hline Do calvings occur at pasture during the summer? & Categorical: Yes, no \\
\hline \multicolumn{2}{|l|}{ Calf management } \\
\hline \multicolumn{2}{|l|}{ Preweaning } \\
\hline Bedding added daily? & Categorical: Yes, no \\
\hline $\begin{array}{l}\text { Frequency of total bedding change or cleaning in unweaned } \\
\text { calf housing }\end{array}$ & Categorical: Never, daily, every other day, weekly or less frequently \\
\hline $\begin{array}{l}\text { Primary method for total bedding change or cleaning in } \\
\text { unweaned calf housing }\end{array}$ & $\begin{array}{l}\text { Categorical: Not cleaned, soiled bedding removed, all bedding removed } \\
\text { and washed with water, all bedding removed and washed with } \\
\text { detergent }\end{array}$ \\
\hline Primary feed for unweaned calves & Categorical: Milk replacer, other \\
\hline Calf feeding hygiene & $\begin{array}{l}\text { Categorical: Utensils not shared, utensils shared and not cleaned, } \\
\text { utensils shared and cleaned between calves }\end{array}$ \\
\hline Cleaning of feeding utensils between feedings & Categorical: Cleaned, not cleaned \\
\hline Calf contacts after separation from dam & $\begin{array}{l}\text { Categorical: Other unweaned animals, weaned animals to } 6 \text { mo of age, } \\
6 \text { mo old to bred, bred heifers, adult cattle, no physical contact }\end{array}$ \\
\hline \multicolumn{2}{|r|}{ (е) } \\
\hline Number of calves from weaning to 6 mo of age & Continuous \\
\hline
\end{tabular}

such animals were present, $80 \%$ of all calves less than 6 mo of age were to be sampled. Fecal samples were also collected from 5 heifers from 6 mo of age until first freshening, 5 lactating cows, and 5 dry cows at each study farm.

At the time of sampling, study personnel collected detailed farm management and demographic data through the administration of a comprehensive management questionnaire. The questionnaire was conducted in the form of an in-person interview with the farm owner or manager. A single member of the study team administered the questionnaire on all farms to eliminate interobserver error. The questionnaire covered a large range of physical and management factors pertaining to each farm (Table 1). Emphasis was placed on factors hypothesized to relate to the prevalence of this parasite, such as colostrum management, nature and frequency of bedding changes, nature of manure management, open or closed herd status, and existence of rodent or wild bird problems.

\section{Sample Processing}

Fecal samples were collected from the rectum of each animal and immediately placed in uniquely labeled screw-topped specimen containers and stored on frozen cold packs while being transported to the laboratory. For the flotation and immunogenic techniques, samples were stored at $4{ }^{\circ} \mathrm{C}$ in the laboratory and processed within 2 wk of collection. A standard quantitative centrifugation concentration flotation technique was initially used on all samples. For each sample, $1 \mathrm{~g}$ of feces was processed using sugar (sg 1.33) as the flotation medium to recover $C$. parvum-like oocysts. Microscopic examination was carried out using bright-field and phase-contrast microscopy. An animal was considered 
positive if an oocyst with the correct morphology (i.e., optical properties, internal structure, size, and shape) was detected in the sample. Criteria used to identify C. parvum-like oocysts included the following: measuring 4 to $6 \mathrm{~mm}$, being spherical with a residuum and sporozoites, refracting pink in sugar, and having a halo in phase. A commercially available ELISA (ProSpecT Cryptosporidium Polyclonal Microplate Assay; AlexonTrend, Inc., Lenexa, KS) was performed on all samples collected from animals $45 \mathrm{~d}$ of age or less.

\section{Nested PCR}

A nested PCR targeting the 18S rRNA gene was used to allow for the differentiation between C. parvum and C. bovis, allowing elucidation of the zoonotic risk posed by cattle within watersheds. All samples testing positive by flotation, ELISA, or both were subjected to the PCR protocol. Additionally, 100 flotation-negative samples were analyzed with this method to determine the degree of agreement between the techniques, as measured by the kappa statistic.

Immediately on return to the laboratory, a 1-mL subsample of feces of approximately 50\% solids was obtained from each individual fecal sample and stored at $-20^{\circ} \mathrm{C}$ in a sealed tube prior to DNA extraction. Upon thawing the feces, we used a modified mechanical disruption method for DNA extraction (Zhu et al., 1998). Samples were examined at the 18S rRNA gene loci using a nested PCR protocol. Two sets of primers amplifying a final fragment of approximately $830 \mathrm{bp}$ were used. The primary reaction consisted of $1 \mu \mathrm{L}$ of $1: 10$ diluted DNA solution obtained from the extraction procedure, added to a mixture consisting of $10.8 \mu \mathrm{L}$ of reverse osmosis water, $2 \mu \mathrm{L}$ of $1 \times$ PCR buffer, $4.8 \mu \mathrm{L}$ of $\mathrm{MgCl}_{2}(50 \mathrm{mM}), 0.4 \mu \mathrm{L}$ of deoxyribonucleoside triphosphates, $0.4 \mu \mathrm{L}$ of the forward primer (SSU 1) $5^{\prime}$ GAT AAC CGT GGT AAT TCT AGA GCTA-3' (10 $\mu M)$, $0.4 \mu \mathrm{L}$ of the reverse primer (SSU 2) 5'- TAA GGT GCT GAA GGA GTA AGG $-3^{\prime}(10 \mu M)$, and $0.2 \mu \mathrm{L}$ of Taq DNA polymerase. The secondary reaction consisted of $1 \mu \mathrm{L}$ of the product from the primary reaction added to a mixture consisting of $13.2 \mu \mathrm{L}$ of water, $2 \mu \mathrm{L}$ of $1 \times$ PCR buffer, $2.4 \mu \mathrm{L}$ of $\mathrm{MgCl}_{2}(50 \mathrm{mM}), 0.4 \mu \mathrm{L}$ of deoxyribonucleoside triphosphates, $0.4 \mu \mathrm{L}$ of the forward nested primer (SSU 3) 5'-GAA RGG TYG TAT TTA TTA GAT AAA GGAAC $-3^{\prime}(10 \mu M), 0.4 \mu \mathrm{L}$ of the reverse nested primer (SSU 4) 5'-AAG GAG TAA GGA ACA ACC TCC A-3' $(10 \mu M)$, and $0.2 \mu \mathrm{L}$ of Taq DNA polymerase. Both the primary and secondary reactions were run under the same conditions: 35 cycles of $96^{\circ} \mathrm{C}$ for $45 \mathrm{~s}, 55^{\circ} \mathrm{C}$ for $45 \mathrm{~s}$, and $72^{\circ} \mathrm{C}$ for $1 \mathrm{~min}$.

\section{DNA Sequencing}

Polymerase chain reaction products were purified using Exonuclease I/Shrimp Alkaline Phosphatase (ExoSAP-IT; USB Corporation, Cleveland, OH). Purified products were sequenced using the internal primers described above in $9-\mu \mathrm{L}$ reactions using an automated sequencer (3730 DNA Analyzer; Applied Biosystems, Foster City, CA). Samples were sequenced in both directions and sequence chromatograms from each strand were aligned and inspected using MEGA 3.1 (Kumar et al., 2004).

\section{Data Management and Statistical Analysis}

Data management, coalition, and validation were performed using Microsoft Access 2000 and Microsoft Excel 2000 (Microsoft, Redbank, CA). The SAS statistical program (Version 9.1 for Windows; SAS Institute, Cary, NC) was used to generate descriptive statistics and manage data. Prevalence was determined as the proportion of samples testing positive for C. parvumlike oocysts relative to those testing negative. Because all samples were analyzed with the flotation method, prevalence is reported based on the results yielded by this method. Prevalence was examined in the population as a whole, at the herd level, and by age.

Fecal flotation results were used in the statistical analyses to identify risk factors associated with the prevalence of $C$. parvum-like oocyst shedding. These analyses were restricted to animals less than $60 \mathrm{~d}$ of age. Such a restriction was used to focus on the population perceived to be at greatest risk, and to increase the power of the statistical analyses. This cut-off was based on the results of a study conducted in an adjacent watershed, which identified an age range of 3 to $60 \mathrm{~d}$ among C. parvum-like-positive animals (mean $15 \mathrm{~d}$, standard deviation $6.6 \mathrm{~d}$; Starkey et al., 2005). To this end, a logistic regression model was used to identify and examine the significance of various risk factors when examined simultaneously. During this process, appropriate transformations, including polynomial transformations, were assessed for continuous variables in an attempt to improve model fit. Individual animal and herd management factors obtained from the management questionnaire were first examined for association with the prevalence using appropriate bivariate techniques. Discrete factors were examined by $\chi^{2}$ test of independence or Fisher's exact test when expected cell counts were less than 5 . Continuous variables were examined by unconditional logistic regression. The model-building step continued with the investigation of correlation among significant variables $(P \leq$ $0.2)$ grouped under each of the management categories (general farm management, maternity management, 
preweaning calf management, postweaning calf management). Pearson correlation coefficients were examined, and when a correlation was found between variables, the decision regarding which to retain was based on biological plausibility. When 3 or more variables were retained in a management category after the above steps, a best subset selection procedure was run, using Mallow's Cp statistic, to determine which to advance to the final model-building step. Age was included as an independent variable in these steps to control for this important risk factor throughout the modelbuilding process. Ultimately, all the significant variables chosen at each management level were submitted, along with age, to a final best subset selection procedure for the selection of the best model of association between parameters of interest and the prevalence of C. parvumlike oocyst shedding.

Because the sampling units (the animals) in this study are clustered into herds, we assumed that this clustering would lead to a correlation in the likelihood of infection within the study population. This correlation between responses occurs because they are dependent on exogenous factors that are associated with these responses (i.e., infection with the organism). Conditioning on an observed set of these factors by controlling for their effect in the analysis and including them as covariates in the logistic regression analysis will sometimes achieve approximate conditional independence. However, more often this correlation in the response arises from both observed and unobserved risk factors. We assumed that the unobserved risk factors were randomly distributed among farms, and the overall significance of this assumption was evaluated by using a mixed-effect logistic regression model (Rosner, 1989). The mixed-effect logistic regression model was specified as follows:

$$
P\left(C P / \alpha, \beta_{i}, \sigma\right)=\frac{1}{\left[1+\exp ^{-\left(\alpha+\Sigma \beta_{i} Z_{i}+\mu_{i} \sigma\right)}\right]}
$$

where $P(C P / \alpha, \beta, \sigma)$ is the probability that an animal within a herd level $\mu_{i}$ would have been shedding $C$. parvum-like oocysts given a set of fixed factors $Z_{i}$ with an effect of $\beta_{i}$. The likelihood ratio test was used to evaluate the significance of the farm random effect parameter in the mixed model. The mixed-effect logistic regression analysis was performed using the EGRET statistical software (Cytel Statistical Software, Cambridge, MA). The effect of each factor on the likelihood of infection with the organism was quantified by the odds ratio (OR), which was computed as the exponent of the respective regression coefficient.

\section{RESULTS}

\section{Descriptive}

A total of 453 samples were collected from animals on the 19 study farms. These samples were obtained from 184 animals of 6 mo of age or less (including 81 animals of $60 \mathrm{~d}$ of age or less), 104 heifers older than 6 mo, and 165 adult animals (lactating and dry cows). There was $92 \%$ agreement between the flotation and the ELISA techniques used in this study; thus, results are reported for the flotation technique because animals of all ages were subjected to this test. Of the $453 \mathrm{sam}$ ples, 16 were determined to be positive by flotation for C. parvum-like oocysts, giving an overall prevalence of $3.53 \%$. This figure is higher than that of $0.9 \%$ reported in a cross-section study previously performed in an adjacent watershed (Wade et al., 2000). When examining herd-level prevalence, $C$. parvum-like oocysts were evident in at least one animal on 8 of the 19 study farms, yielding a herd-level prevalence of $42 \%$. Prevalence among animals sampled on the study farms ranged from 3.1 to $21.4 \%$.

The average age of animals shedding oocysts was 16 $\mathrm{d}$, with a standard deviation of $9.2 \mathrm{~d}$ and a range of 3 to $31 \mathrm{~d}$. When examining animals less than or equal to $60 \mathrm{~d}$ of age, prevalence was found to be $20 \%$ across the study subjects. The prevalence was higher still when animals of $31 \mathrm{~d}$ of age or younger were examined, with $32 \%$ of such animals testing positive at the time of sampling.

\section{Risk Factors}

After variables within in each management category were examined using bivariate techniques and significant variables were investigated with Pearson correlation coefficients, several variables remained and were subsequently analyzed using a best subset selection procedure (Table 2 ). The variables under consideration during the development of the final best subset model were age, type of water supply for the barn, type of housing for milking cows, nature of calf housing (tied vs. not tied), total number of unweaned calves on a farm, and contact of postweaned calves with adult cows (binary).

The factors that were ultimately chosen for the multivariate investigation of association with the prevalence of $C$. parvum-like oocyst shedding were age, source of barn water, calf housing (tied vs. not tied), and number of unweaned stock on the farm. The results of the logistic regression based on these parameters, including parameter estimates, $\mathrm{OR}$, and confidence intervals, can be seen in Table 3. None of the polynomial transformations were significant. The likelihood of shedding oocysts de- 
Table 2. Significant variables ${ }^{1}$ as identified by bivariate analysis, after accounting for correlation patterns, grouped by management categories

\begin{tabular}{lll}
\hline Management category & Variable & $P$-value \\
\hline General farm management & Age & 0.0137 \\
& Barn water source & 0.133 \\
& Barn type & 0.0033 \\
Maternity management & Milking cows allowed access to pasture during summer & 0.185 \\
Preweaning calf management & Not significant & \\
& Number of unweaned calves at the time of the study & 0.0134 \\
& Calf housing (tied vs. not tied) & 0.165 \\
& Calf housing (greenhouse vs. not greenhouse) & 0.0836 \\
& Contact with older calves (weaned to 6 mo) & 0.0807 \\
& Frequency of total bedding change & 0.0077 \\
Postweaning calf management & Summer provision of water to calves & 0.0818 \\
\hline
\end{tabular}

${ }^{1}$ Significant at $P \leq 0.2$.

creased as the source of barn water changed from nonwell to well sources $(\mathrm{OR}=0.02)$ and as the age of the animal increased $(\mathrm{OR}=0.9)$. Calves housed in tie stalls were at greater risk of shedding the protozoa than those in other forms of housing, such as greenhouses, individual pens, or group pens. The likelihood of shedding C. parvum-like oocysts increased with the number of unweaned calves in the herd (Table 3). Investigation of the hierarchical nature of the data set was performed using a mixed-effect logistic regression model. This analysis indicated that there was no significant correlation of risk by group among the study subjects, as indicated by a nonsignificant random effect parameter (data not shown).

\section{PCR and Sequencing Results}

Of the 16 flotation-positive samples, 12 were successfully amplified using the nested PCR. An additional 100 flotation-negative samples were analyzed with the PCR protocol to determine the degree of agreement between the 2 techniques. Statistical analysis of all samples examined by PCR and flotation indicated a kappa statistic of 0.71 , a level considered to be associated with a high degree of association above that expected by chance alone (Fleiss, 1981). Subsequent DNA sequence analysis of the PCR-positive samples indicated that $50 \%$ of the isolates were $C$. bovis, a nonzoonotic Cryptosporidium species (Fayer et al., 2005).

\section{DISCUSSION}

The study area forms part of the headwaters of the Susquehanna River, the source of water for several downstream communities, including the City of Binghamton. Additionally, the river provides a large proportion of Chesapeake Bay's fresh water, an area of increasing environmental concern and home to extensive fishing and to crustacean and bivalve industries (Fayer et al., 2002). Dairy cattle have been implicated as the source of drinking water contamination with $C$. parvum, although evidence to this effect is not entirely conclusive (Xiao and Ryan, 2004). Therefore, quantification of the prevalence of $C$. parvum infection and identification of associated risk factors among dairy cattle in watersheds remains an important undertaking.

Table 3. Results of the logistic regression analysis for the association between putative risk factors and odds of infection with Cryptosporidium parvum

\begin{tabular}{lllll}
\hline Factor & $\begin{array}{l}\text { Regression } \\
\text { coefficient }\end{array}$ & $\begin{array}{l}\text { Standard } \\
\text { error }\end{array}$ & $\begin{array}{l}\text { Estimated } \\
\text { odds } \\
\text { ratio }\end{array}$ & $\begin{array}{l}95 \% \\
\text { confidence } \\
\text { interval }\end{array}$ \\
\hline $\begin{array}{l}\text { Age } \\
\text { Barn water source }\end{array}$ & -0.105 & 0.0353 & & \\
$\quad$ Well & -3.90 & 1.335 & 0.020 & $0.001,0.285$ \\
$\quad$ Non-well & 0 & & 1 & \\
$\quad$ Calf housing & 3.77 & 1.26 & 43.4 & $3.66,515$ \\
$\quad \begin{array}{l}\text { Tied } \\
\text { Not tied }\end{array}$ & 0 & 0.125 & & \\
$\begin{array}{l}\text { Number of unweaned calves } \\
\text { Intercept }\end{array}$ & 0.383 & 1.70 & & \\
\hline
\end{tabular}


It is also important that future studies consider the role of $C$. bovis. As reported in this study and elsewhere in the literature (Santin et al., 2004), up to $50 \%$ of isolates that would have been diagnosed as C. parvum via traditional methods (i.e., C. parvum-like) are in fact C. bovis, a nonzoonotic species of Cryptosporidium.

The overall prevalence of 3.9\% observed in this study via the flotation method is higher than the $0.9 \%$ reported in a cross-sectional study conducted in an adjacent watershed (Wade et al., 2000). Although similar age stratification was used in both study designs, additional attention was paid to the youngest animals within the less than 6-mo-old category in the present study, potentially explaining the increase in observed prevalence. The prevalence of $C$. parvum-like oocyst shedding among unweaned animals in this study was $20 \%$, a level significantly higher than the overall prevalence and in keeping with other reports in the literature that this age group has the highest prevalence of the organism (Santin et al., 2004). The present study identified a relatively high herd-level prevalence of the organism of $42 \%$. This finding is also in keeping with previous reports in the literature (Garber et al., 1994; Wade et al., 2000).

As indicated by the kappa statistic of 0.71 , there was a high degree of agreement between the flotation and the PCR methods used in this study. For the purposes of reporting prevalence and investigating risk factors in the present study, we chose to use the results of flotation. This decision was based on the fact that all samples were subjected to this test and thus all had an equal chance of becoming positive. Additionally, this test remains in common use among practitioners and in diagnostic laboratories throughout the United States. This decision subsequently precluded the investigation of factors that would have led to a differential prevalence of $C$. parvum and C. bovis in the present study. Such work would require a larger number of positive animals to draw conclusions, and could initially be performed as a case-control study or a larger retrospective cross-sectional study.

For the purpose of model building, we focused our analysis on animals that were less than $60 \mathrm{~d}$ of age. The rationale for this strategy is 2-fold. First, from our previous studies we learned that among the populations of cattle in New York State, the risk of infection is limited to younger calves that are typically less than $60 \mathrm{~d}$ of age (Wade et al., 2000; Starkey et al., 2005). Second, other researchers found similar results in other cattle populations outside New York State (Santin et al., 2004). Given these findings and the potential for increased statistical power, we opted to restrict the analysis to cattle below $60 \mathrm{~d}$ of age.
Among the findings of interest in the final logistic regression model was the estimated $37 \%$ increase in the odds of infection among those animals whose barn water source was not a well. In the study population, non-well sources of water were either springs or streams. It is possible that these water sources are associated with an increased prevalence because they may have a greater risk of fecal contamination relative to well water. In the final model, there was also a significant increase in the risk of infection in calves that were housed in a tied manner, with a point estimate indicating a $434 \%$ increase in the odds of infection. Most other unweaned calves in the study population were housed in greenhouses. One possible explanation for this increased risk among tied animals is the relative ease of contact with immediate neighbors in most tied environments, thereby increasing the risk of fecal-oral contact. However, it is important to note that even moderate physical separation may not eliminate the risk of transmission of this organism owing to the frequently propulsive nature of the diarrhea associated with the infection. The number of unweaned animals on a property was significantly associated with an increase in the risk of C. parvum-like oocyst shedding. This finding is logical, because an increased number of at-risk (i.e., unweaned) animals would be expected to increase both the chances of detecting infection and the likelihood of an uninfected animal encountering an infected animal or infectious oocyst in its environment.

The study was designed to increase both internal and external validity; however, a large-scale longitudinal study needs to be conducted to confirm the identity of the identified risk factors and to allow causal inferences to be made. Considering increasing evidence regarding the relatively high prevalence of $C$. bovis, further work is also needed to better quantify the risk cattle pose to water supplies and downstream water users. An integrative approach combining stochastic and deterministic reasoning with data in the literature may aid in identifying a useful determination of risk.

\section{ACKNOWLEDGMENTS}

Many organizations and individuals contributed to the success of this project, including the Water Resource Institute at Cornell University, the Delaware County Soil and Water Conservation district, and the students and staff of the Parasitology Section at Cornell University's College of Veterinary Medicine. This work was partially supported by a grant from the federal Environmental Protection Agency (grant \#403) and from the USDA-Cooperative State Research, Education, and Extension Service program (grant \#2002-35212-12317). 


\section{REFERENCES}

Current, W. L., N. C. Reese, J. V. Ernst, W. S. Bailey, M. B. Heyman, and W. M. Weinstein. 1983. Human cryptosporidiosis in immunocompetent and immunodeficient persons. Studies of an outbreak and experimental transmission. N. Engl. J. Med. 308:1252-1257.

Fayer, R., U. Morgan, and S. J. Upton. 2000. Epidemiology of Cryptosporidium: Transmission, detection and identification. Int. J. Parasitol. 30:1305-1322.

Fayer, R., J. M. Trout, E. J. Lewis, L. Xiao, A. Lal, M. C. Jenkins, and T. K. Graczyk. 2002. Temporal variability of Cryptosporidium in the Chesapeake Bay. Parasitol. Res. 88:998-1003.

Fayer, R., M. Santin, and L. Xiao. 2005. Cryptosporidium bovis $\mathrm{n}$. sp. (Apicomplexa: Cryptosporidiidae) in cattle (Bos taurus). J. Parasitol. 91:624-629.

Fleiss, J. L. 1981. Statistical Methods for Rates and Proportions. 2nd ed. John Wiley \& Sons, New York, NY.

Garber, L. P., M. D. Salman, H. S. Hurd, T. Keefe, and J. L. Schlater. 1994. Potential risk factors for Cryptosporidium infection in dairy calves. J. Am. Vet. Med. Assoc. 205:86-91.

Glaberman, S., J. E. Moore, C. J. Lowery, R. M. Chalmers, I. Sulaiman, K. Elwin, P. J. Rooney, B. C. Millar, J. S. G. Dooley, A. A. Lal, and L. Xiao. 2002. Three drinking-water-associated cryptosporidiosis outbreaks, Northern Ireland. Emerg. Infect. Dis. 8:631-633.

Hunter, P. R., and R. C. Thompson. 2005. The zoonotic transmission of Giardia and Cryptosporidium. Int. J. Parasitol. 35:1181-1190.

Kumar, S., K. Tamura, and M. Nei. 2004. MEGA3: Integrated software for molecular evolutionary genetics analysis and sequence alignment. Brief. Bioinform. 5:150-163.

Mac Kenzie, W. R., N. J. Hoxie, M. E. Proctor, M. S. Gradus, K. A. Blair, D. E. Peterson, J. J. Kazmierczak, D. G. Addiss, K. R. Fox, J. B. Rose, and J. P. Davis. 1994. A massive outbreak in Milwaukee of Cryptosporidium infection transmitted through the public water supply. N. Engl. J. Med. 331:161-167.

Maggi, P., A. Larocca, M. Quarto, G. Serio, O. Brandonisio, and G. Angarano. 2000. Effect of antiretroviral therapy on cryptosporidiosis and microsporidiosis in patients infected with human immunodeficiency virus type 1. Eur. J. Clin. Microbiol. Infect. Dis. $19: 213-217$.

Morgan-Ryan, U. M., A. Fall, L. A. Ward, N. Hijjawi, I. Sulaiman, R. Fayer, R. C. Thompson, M. Olson, A. Lal, and L. Xiao. 2002. Cryptosporidium hominis n.sp. (Apicomplexa: Cryptosporidiidae) from Homo sapiens. J. Eukary. Micro. 49:433-440.

Naciri, M., M. P. Lefay, R. Mancassola, P. Poirier, and R. Chermette. 1999. Role of Cryptosporidium parvum as a pathogen in neonatal diarrhoea complex in suckling and dairy calves in France. Vet. Parasitol. 85:245-257.

Nannini, E. C., and P. C. Okhuysen. 2002. HIV1 and the gut in the era of highly active antiretroviral therapy. Curr. Gastroenterol. Rep. 4:392-398.
Navin, T. R., and D. D. Juranek. 1984. Cryptosporidiosis: Clinical, epidemiologic, and parasitologic review. Rev. Infect. Dis. 6:313-327.

New York State Department of Agriculture and Markets. 2006. Subject: Ag Facts. http://www.agmkt.state.ny.us/agfacts.html Accessed Apr. 11, 2006.

Okhuysen, P. C., C. L. Chappell, J. H. Crabb, C. R. Sterling, and H. L. DuPont. 1999. Virulence of three distinct Cryptosporidium parvum isolates for healthy adults. J. Infect. Dis. 180:1275-1281.

Ong, C. S. L., D. L. Eisler, S. H. Goh, J. Tomblin, F. M. Awad-ElKariem, C. B. Beard, L. Xiao, I. Sulaiman, A. Lal, M. Fyfe, A. King, W. R. Bowie, and J. L. Isaac-Renton. 1999. Molecular epidemiology of cryptosporidiosis outbreaks and transmission in British Columbia, Canada. Am. J. Trop. Med. Hyg. 61:63-69.

Panciera, R. J., R. W. Thomassen, and F. M. Garner. 1971. Cryptosporidial infection in a calf. Vet. Pathol. 8:479-484.

Patel, S., S. Pedraza-Diaz, J. McLauchlin, and D. P. Casemore. 1998. Molecular characterisation of Cryptosporidium parvum from two large suspected waterborne outbreaks. Outbreak Control Team South and West Devon 1995, Incident Management Team and Further Epidemiological and Microbiological Studies Subgroup North Thames 1997. Commun. Dis. Public Health 1:231-233.

Rosner, B. 1989. Multivariate methods for clustered binary data with more than one level of nesting. J. Am. Stat. Assoc. 84:373-380.

Santin, M., J. M. Trout, L. Xiao, L. Zhou, E. Greiner, and R. Fayer. 2004. Prevalence and age-related variation of Cryptosporidium species and genotypes in dairy calves. Vet. Parasitol. 122:103117.

Starkey, S. R., S. E. Wade, S. Schaaf, and H. O. Mohammed. 2005. Incidence of Cryptosporidium parvum in the dairy cattle population in a New York City watershed. Vet. Parasitol. 131:197-205.

Sulaiman, I. M., A. A. Lal, and L. Xiao. 2001. A population genetic study of the Cryptosporidium parvum human genotype parasites. J. Eukaryot. Microbiol. (Suppl.):24S-27S.

Tumwine, J. K., A. Kekitiinwa, S. Bakeera-Kitaka, G. Ndeezi, R. Downing, X. Feng, D. E. Akiyoshi, and S. Tzipori. 2005. Cryptosporidiosis and microsporidiosis in Ugandan children with persistent diarrhea with and without concurrent infection with the human immunodeficiency virus. Am. J. Trop. Med. Hyg. 73:921-925.

Wade, S. E., H. O. Mohammed, and S. L. Schaaf. 2000. Prevalence of Giardia sp., Cryptosporidium parvum and Cryptosporidium muris (C. andersoni) in 109 dairy herds in five counties of southeastern New York. Vet. Parasitol. 93:1-11.

Xiao, L., and U. M. Ryan. 2004. Cryptosporidiosis: An update in molecular epidemiology. Curr. Opin. Infect. Dis. 17:483-490.

Zhu, G., M. J. Marchewka, J. G. Ennis, and J. S. Keithly. 1998. Direct isolation of DNA from patient stools for polymerase chain reaction detection of Cryptosporidium parvum. J. Infect. Dis. 177:14431446. 